Supporting Information

\title{
Hydrogen/Deuterium Exchange Measurements May Provide an Incomplete View of Protein Dynamics: A Case Study on \\ Cytochrome $c$
}

\author{
Pablo M. Scrosati, Victor Yin, and Lars Konermann* \\ Department of Chemistry, The University of Western Ontario, London, Ontario \\ N6A 5B7, Canada
}

* corresponding author: konerman@uwo.ca

This file contains:

Complete Methods Section

Figure S1: UV-Vis and MS characterization of cyt $c$ samples.

Figure S2: HDX sequence coverage.

Figure S3: Time dependence of various interatomic distances during MD runs.

Figure S4: Dynamic H-bond patterns of different cyt $c$ conformers.

Figure S5: Summary of backbone nitrogen RMSF values.

DynamX-generated kinetic data (in a separate MS Excel file). 
Protein and Reagents. Sodium phosphate, $\mathrm{HCl}, \mathrm{D}_{2} \mathrm{O}(>99 \%)$, and equine cyt $c$ were purchased from Sigma (St. Louis, MO). The protein obtained from this source is in the Fe(III) state. ${ }^{1}$ To eliminate possible trace amounts of $\mathrm{Fe}(\mathrm{II})$ cyt $c, 30 \mu \mathrm{M}$ cyt $c$ in $50 \mathrm{mM}$ sodium phosphate buffer ( $\mathrm{pH} 7$ ) was exposed to $4 \mathrm{mM}$ of the oxidizing agent potassium ferricyanide for $5 \mathrm{~min} .^{2} \mathrm{The} \mathrm{Fe}(\mathrm{III})$ cyt $c$ was buffer exchanged three times using Amicon $10 \mathrm{kDa}$ centrifuge-filters at $13000 \mathrm{~g}$ for 15 min. The protein was recovered in $50 \mathrm{mM}$ sodium phosphate at $\mathrm{pH} 7$, flash frozen in liquid nitrogen and stored at $-80{ }^{\circ} \mathrm{C}$. $\mathrm{Fe}(\mathrm{II})$ cyt $c$ was prepared in identical fashion, except that potassium ferricyanide was substituted for the reducing agent sodium ascorbate. ${ }^{3} \mathrm{UV}$-visible spectroscopy of the two types of samples generated the expected absorption signatures (Figure S1A). ${ }^{4}$ These spectra remained unchanged over the course of 100 min HDX measurements. MS confirmed that ferricyanide and ascorbate treatment did not cause any covalent modifications (Figure S1B).

Hydrogen-Deuterium Exchange Mass Spectrometry. HDX was performed at $22 \pm 1^{\circ} \mathrm{C}$ by adding $15 \mu \mathrm{L}$ of $30 \mu \mathrm{M}$ cyt $c$ to $180 \mu \mathrm{L} \mathrm{D}{ }_{2} \mathrm{O}$ buffered with $50 \mathrm{mM}$ sodium phosphate, resulting in $92 \% \mathrm{D}_{2} \mathrm{O}$. The $\mathrm{pH}-$ meter reading was 6.6 which corresponds to $\mathrm{pD} 7.0 .^{5}$ Aliquots were removed at various time points between 0.2 and $100 \mathrm{~min}$. These aliquots $(195 \mu \mathrm{L})$ were quenched by the addition of $5 \mu \mathrm{L} 1.9 \mathrm{M} \mathrm{HCl}$, corresponding to $\mathrm{pH} 2.5$. The quenched aliquots were flash frozen and stored in liquid nitrogen until further analysis. The final protein concentration was $2.25 \mu \mathrm{M}$. Additional $m_{0}$ and $m_{100}$ control samples served to correct for artifactual deuterium in-exchange and back-exchange, respectively. $m_{0}$ samples were prepared by mixing $15 \mu \mathrm{L}$ cyt $c$ into quenched $\mathrm{D}_{2} \mathrm{O}$ buffer. $m_{100}$ samples were prepared by exposing cyt $c$ state to quenched $\mathrm{D}_{2} \mathrm{O}$ buffer for 48 hours. For mass analysis, frozen aliquots were quickly thawed to $\sim 0{ }^{\circ} \mathrm{C}$ and injected into a nanoACQUITY HDX/UPLC system (Waters, Milford, MA). The protein was digested online 
using an Enzymate BEH pepsin column for two minutes at $12{ }^{\circ} \mathrm{C}$. Peptides were trapped and desalted on a BEH C18 guard column, and separated on a BEH C18 50 mm column using a 10 min water/acetonitrile gradient at $0{ }^{\circ} \mathrm{C}$, followed by $10 \mathrm{~min}$ re-equilibration. Blank gradients were run between samples to eliminate carryover. The column outflow was analyzed on a Waters Synapt G2-Si ESI Q-TOF mass spectrometer. Peptides were identified using tandem MS and ProteinLynx software, followed by manual validation. No high $\mathrm{S} / \mathrm{N}$ peptides were found for the region ${ }^{12} \mathrm{QKCAQCH}{ }^{18}$ which contains the covalently bound heme. ${ }^{6}$ This left 61 detected peptides for a sequence coverage of 93.3\% (Figure S2A). Data analysis was performed using DynamX 3.0. HDX profiles for peptides covering similar regions were highly consistent. The data analysis below therefore focuses on a subset of 12 peptides that had the highest $\mathrm{S} / \mathrm{N}$ and provides the same coverage as the complete set (Figure S2B). HDX occurred in the EX2 regime, seen from unimodal isotope distributions that shifted to higher mass with increasing deuteration time. HDX profiles were normalized to percent deuteration according to $\% D=\left(m_{t}-m_{0}\right) /\left(m_{100}-m_{0}\right)$, where $m_{t}$ is the centroid $\mathrm{m} / \mathrm{z}$ for time $t$. Samples were prepared and experiments were conducted in triplicate; error bars correspond to standard error of the mean.

Molecular Dynamics Simulations. All-atom MD simulations were conducted using GROMACS 2020.3 with GPU acceleration, ${ }^{7}$ with the CHARMM36 force field ${ }^{8}$ and TIP3P water. ${ }^{9}$ These conditions were chosen on the basis of their proven performance for investigations on protein folding and dynamics. ${ }^{10}$ Using the PDB file $1 \mathrm{HRC}^{6}$ as starting coordinates, a cubic simulation box with periodic boundary conditions, containing one protein and $\sim 10000$ waters was set up, with a minimum distance of $1 \mathrm{~nm}$ between the protein and box boundaries. Atomic charges for sixcoordinate $\mathrm{Fe}(\mathrm{III})$ and $\mathrm{Fe}(\mathrm{II})$ heme $c$ were taken from ref. ${ }^{11}$, with covalent bonds from $\mathrm{Fe}$ to $\mathrm{Me} 80$ 
$\mathrm{S}$ and Fe to His $18 \mathrm{~N} \varepsilon$. Most parameters for 5-coordinate Fe(II) heme (without M80-Fe covalent bond) were adopted from $b$-type heme in CHARMM $36 .{ }^{8}$ Partial charges for heme core atoms were used as is, while partial charges of peripheral atoms such as those in heme propionates were taken from ref. ${ }^{11}$ From these parameters, 5 -coordinate Fe(III) heme was generated by uniformly scaling atomic charges of porphyrin core atoms to increase the net charge by 1.0. For modeling 4coordinate $\mathrm{Fe}(\mathrm{III})$ heme we used the same atomic charges as for the 5 -coordinate $\mathrm{Fe}(\mathrm{III})$ state, but lacking both axial bonds. With default titration patterns, the overall protein charge was $7+$ for $\mathrm{Fe}(\mathrm{III}) \mathrm{cyt} c$ and $6+$ for $\mathrm{Fe}(\mathrm{II}) \mathrm{cyt} c$. Excess $\mathrm{Cl}^{-}$were added to ensure charge neutrality. Following energy minimization and NPT equilibration for $1 \mathrm{~ns}, 1 \mu \mathrm{s}$ NPT production runs were performed with a 2 fs time step. For simulations on 4-coordinate Fe(III) cyt $c$, an additional $100 \mathrm{~ns}$ equilibration was performed prior to production runs. Temperature and pressure coupling (298 K and 1 bar) used the Nosé-Hoover thermostat and Parrinello-Rahman barostat, respectively. Covalent bonds between heavy atoms and hydrogens were constrained using the LINCS algorithm (this did not include H-bonds; these noncovalent contacts were allowed to open/close in response to protein fluctuations). Short-range Coulomb and van der Waals cutoffs were set to $1 \mathrm{~nm}$. Longrange electrostatics were treated via Particle Mesh Ewald summation. Time-dependent properties were averaged over three independent $1 \mu$ s production runs for each of the four protein states. $\mathrm{H}$ bonded NH sites were initially identified using the gmx hbond routine $^{7}$ by scanning each backbone $\mathrm{NH}$ against all $\mathrm{CO}$ sites for all time points, using a $\mathrm{NO}$ distance $<0.35 \mathrm{~nm}$ and a $150^{\circ}-180^{\circ} \mathrm{N}-$ $\mathrm{H}-\mathrm{O}$ angle as inclusion criteria. A few $\mathrm{NH}$ sites never engaged in H-bonding for any of the protein states examined here (E62, A83, and T89). Fe(II) cyt $c$ did not have H-bonding for D2, V20, E21, K22, F36, K39, G41, T58, E61, E62, K79, A83, K87, K88, and T89. As a consequence, the "Fe(III) minus $\mathrm{Fe}(\mathrm{II}) "$ difference plots in Figure 6 do not show data points for these residues. 
Opening/closing of $\mathrm{NH}$ sites that were $\mathrm{H}$-bonded at least some of the time was tracked by monitoring the $\mathrm{H} \cdots \mathrm{O}$ distance with a value $<0.25 \mathrm{~nm}$ as criterion for an intact $\mathrm{H}$-bond. ${ }^{12}$ 

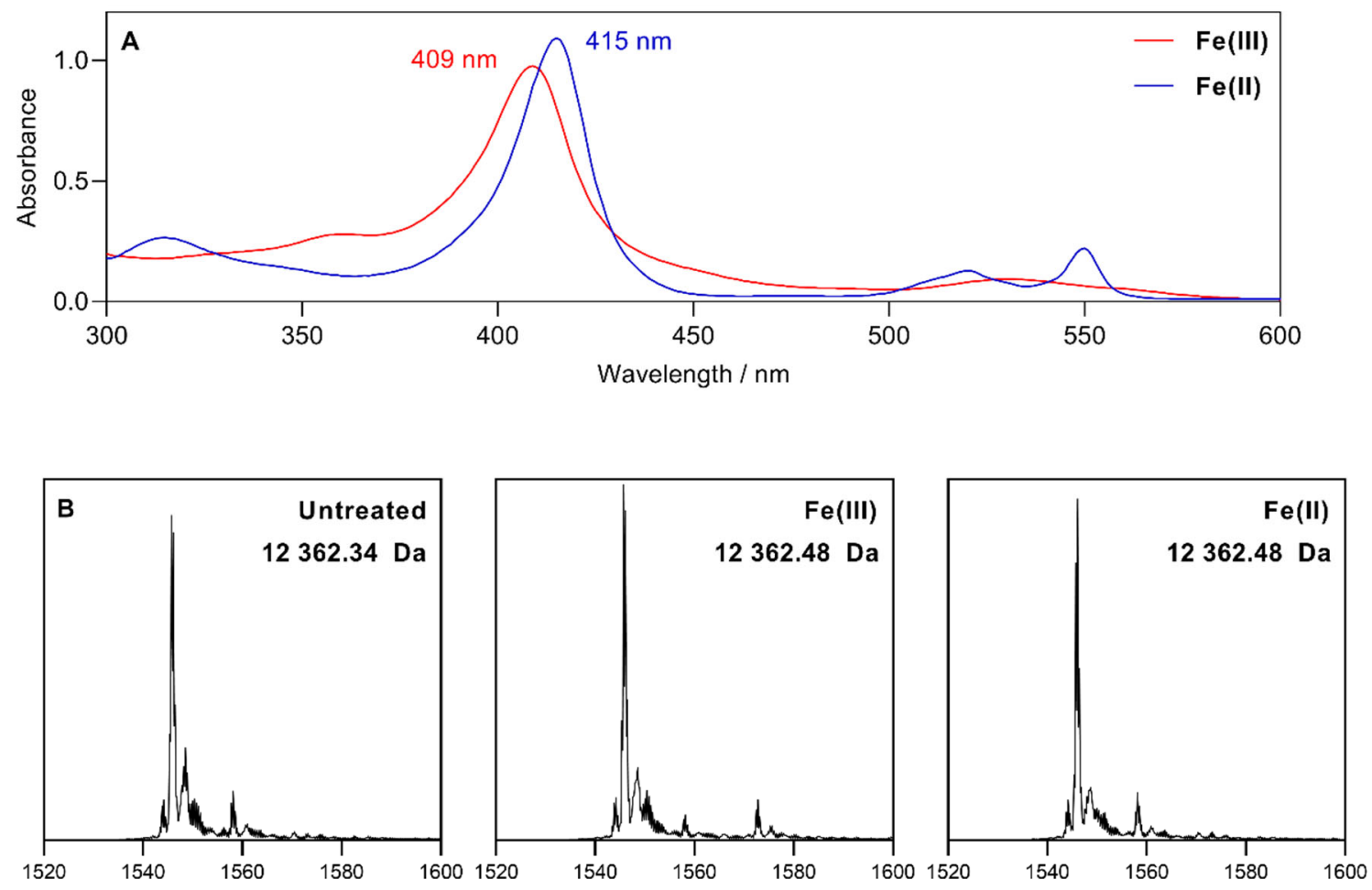

Figure S1. (A) UV-Vis spectra of Fe(III) and Fe(II) cyt $c$. Fe(III) cyt $c$ showed a Soret maximum at $409 \mathrm{~nm}$ and a single Q band at $528 \mathrm{~nm}$. Fe(II) cyt $c$ spectra had a Soret maximum at $415 \mathrm{~nm}$, a $\beta$-band at $520 \mathrm{~nm}$, and an $\alpha$-band at $550 \mathrm{~nm}$. (B) Intact protein ESI mass spectra (showing the $8+$ charge state) after solvent exchange into $50 \mathrm{mM}$ aqueous ammonium acetate. 
A

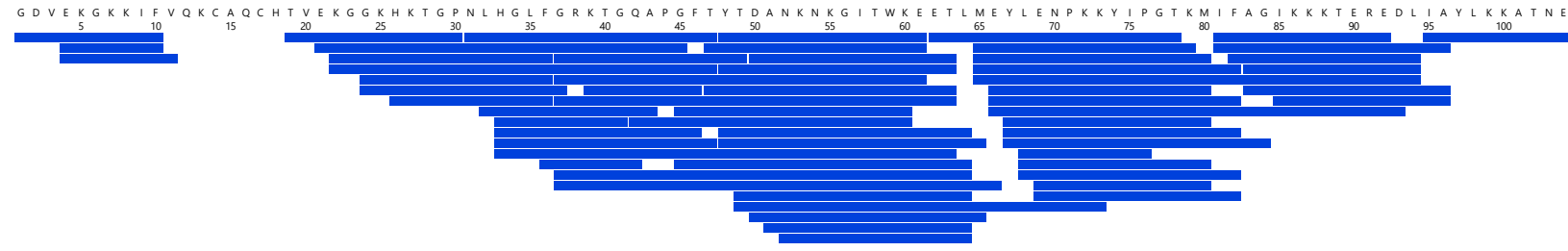

B

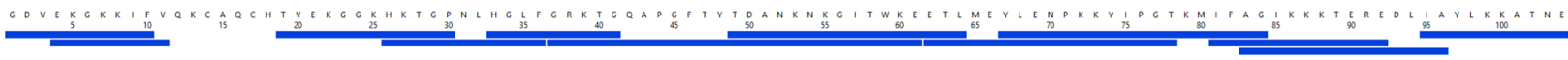

Figure S2. (A) DynamX peptide coverage map with 61 detected peptides, for a sequence coverage of $93.3 \%$. (B) Simplified subset of 12 peptides that provides the same sequence coverage. 


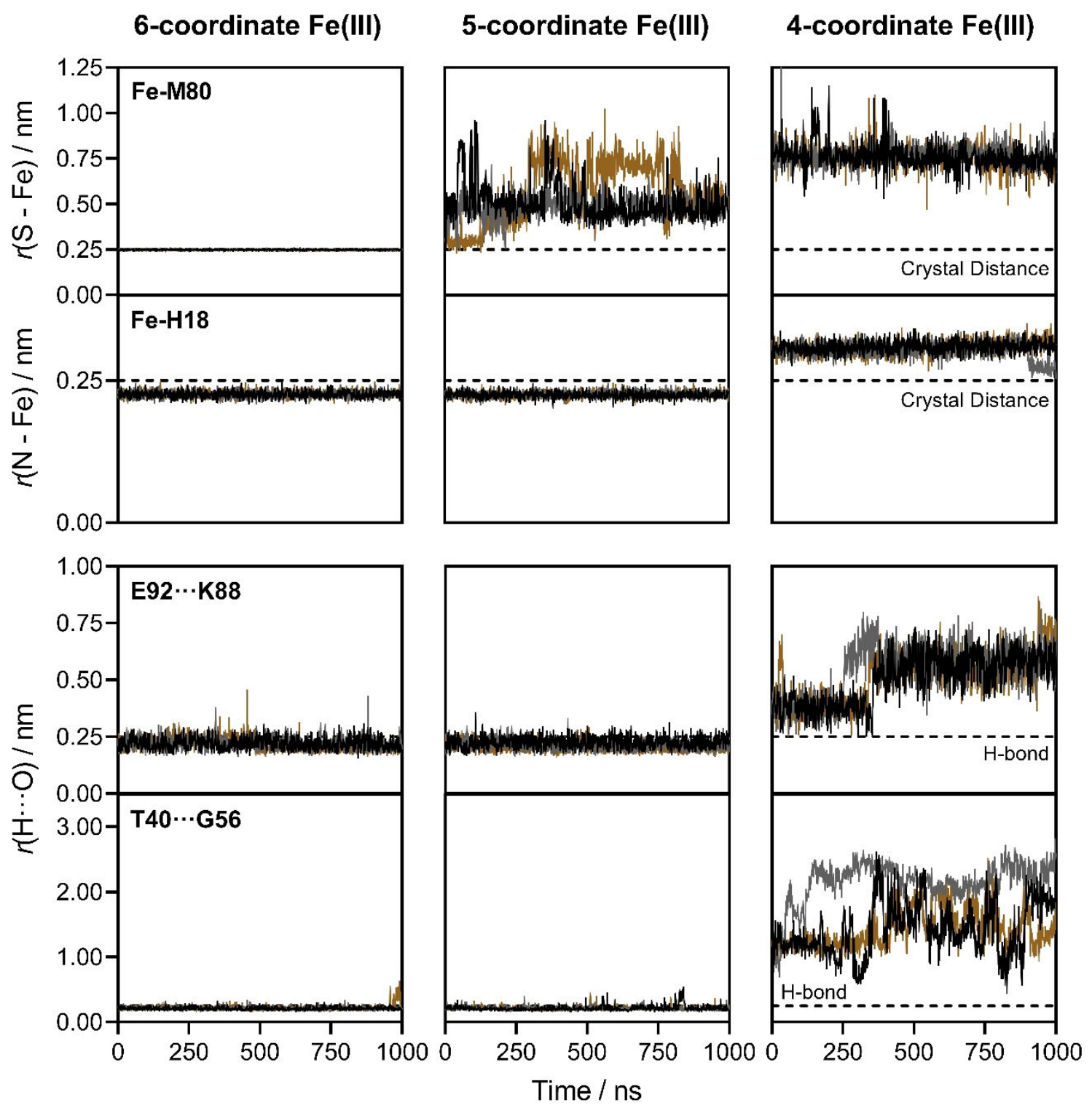

Figure S3. Interatomic distances seen during MD runs. Column on the left: six-coordinate M80$\mathrm{Fe}(\mathrm{III})-\mathrm{H} 18$. Center column: 5-coordinate Fe(III)-M80. Column on the right: 4-coordinate Fe(III). Each panel contains data for three $1 \mu \mathrm{s}$ MD runs. Top row: Distal heme coordination. Second row: Proximal heme coordination. Third row: Example H-bond contact on the distal side. Fourth row: Example H-bond contact on the proximal side. 
Residue

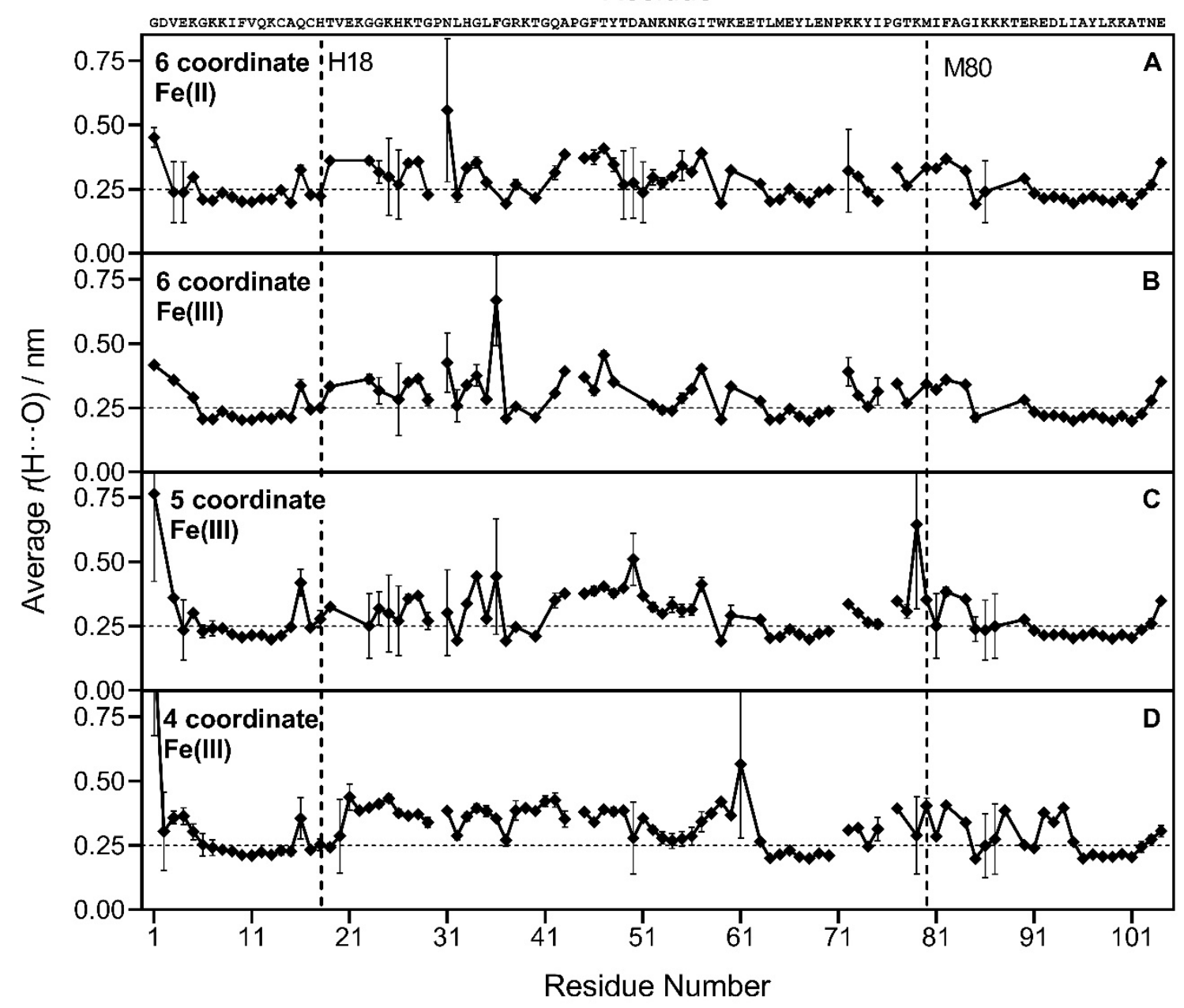

Figure S4. MD-derived H-bond dynamics for different heme ligation states: (A) Native Fe(II) cyt c, (B) 6-coordinate M80-Fe(III)-H18, (C) 5-coordinate Fe(III)-M80, and (D) 4-coordinate Fe(III). The panels show the distance between backbone NH hydrogen and OC oxygen, averaged over three independent $1 \mu \mathrm{s}$ runs for each condition. Values below $0.25 \mathrm{~nm}$ (horizontal dashed lines) indicate intact $\mathrm{H}$-bonds. NH sites undergoing extensive opening/closing are characterized by large error bars (standard error of the mean). Pro residues appear as gaps. 


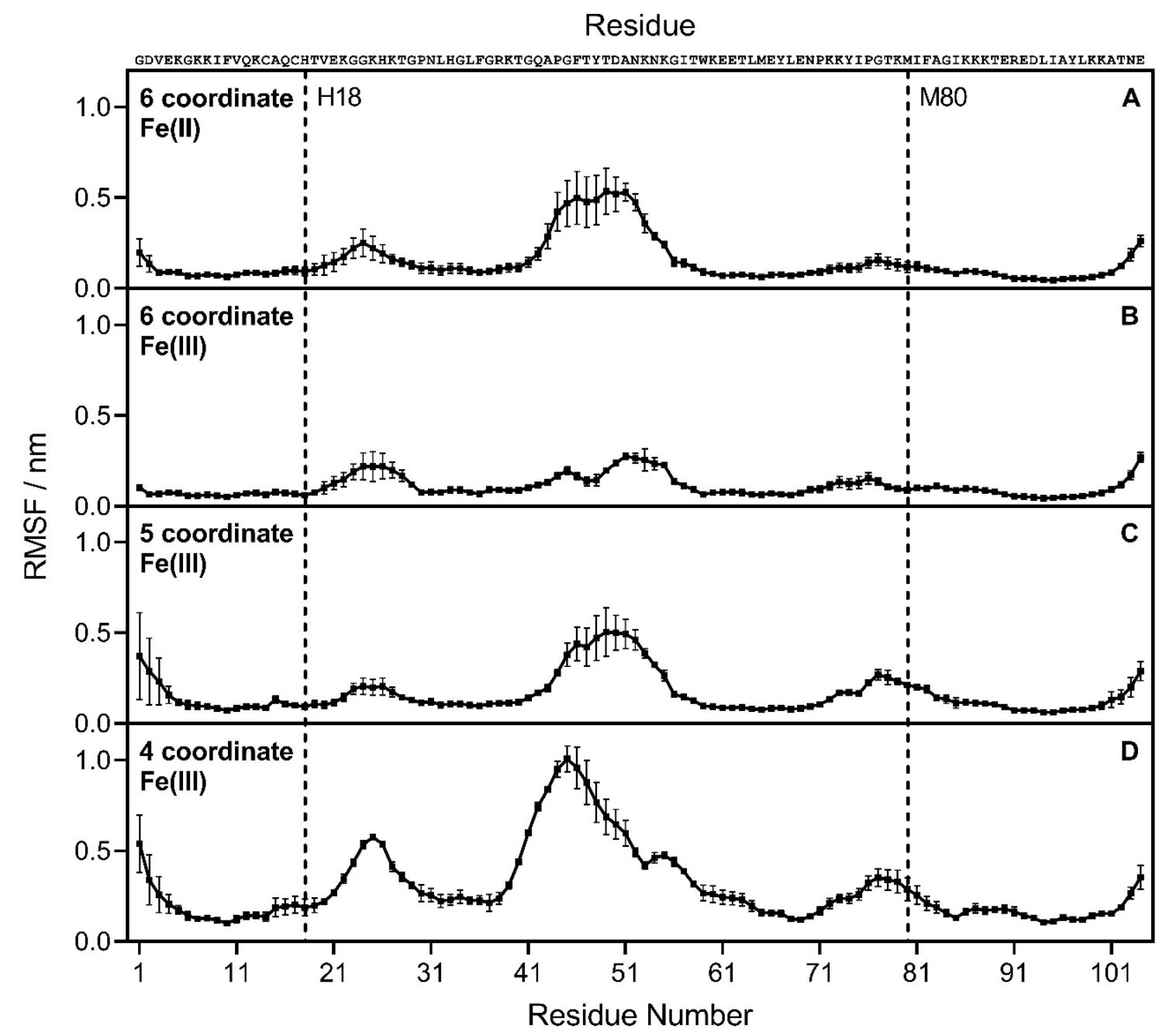

Figure S5. MD-derived root mean square fluctuation (RMSF) of backbone $\mathrm{N}$ atoms around their average position. These data directly reflect the extent of structural fluctuations for each of the atoms. (A) Native Fe(II) cyt c, (B) six-coordinate M80-Fe(III)-H18, (C) 5-coordinate Fe(III)-M80, and (D) 4-coordinate Fe(III). The data were averaged over three independent $1 \mu$ s runs for each condition. Error bars (SEM) indicate the deviations between individual runs. 


\section{SI References}

(1) Yin, V.; Shaw, G. S.; Konermann, L. Cytochrome c as a Peroxidase: Activation of the PreCatalytic Native State by H2O2-Induced Covalent Modifications. J. Am. Chem. Soc. 2017, 139, 15701-15709.

(2) Brandt, K. G.; Parks, P. C.; Czerlinski, G. H.; Hess, G. P. On the Elucidation of the pH Dependence of the Oxidation-Reduction Potential of Cytochrome c at Alkaline pH. J. Biol. Chem. 1966, 241, 4180-+.

(3) Myer, Y. P.; Kumar, S. Ascorbate Reduction of Horse Heart Cytochrome c. J. Biol. Chem. 1984, 259, 8144-8150.

(4) Margoliash, E.; Frohwirt, N. Spectrum of horse-heart cytochrome c. Biochem. J. 1959, 71, 570-572.

(5) Englander, S. W.; Mayne, L.; Kan, Z. Y.; Hu, W. B. Protein Folding-How and Why: By Hydrogen Exchange, Fragment Separation, and Mass Spectrometry. Annu. Rev. Biophys. 2016, 45, 135-152.

(6) Bushnell, G. W.; Louie, G. V.; Brayer, G. D. High-resolution Three-dimensional Structure of Horse Heart Cytochrome c. J. Mol. Biol. 1990, 214, 585-595.

(7) Abraham, M. J.; Murtola, T.; Schulz, R.; Páll, S.; Smith, J. C.; Hess, B.; Lindahl, E. GROMACS: High performance molecular simulations through multi-level parallelism from laptops to supercomputers. SoftwareX 2015, 1-2, 19-25.

(8) Huang, J.; MacKerell, A. D. CHARMM36 all-atom additive protein force field: Validation based on comparison to NMR data. J. Comput. Chem. 2013, 34, 2135-2145.

(9) Jorgensen, W. L.; Chandrasekhar, J.; Madura, J. D.; Impey, R. W.; Klein, M. L. Comparison of simple potential functions for simulating liquid water. J. Chem. Phys. 1983, 79, 926-935.

(10) Piana, S.; Lindorff-Larsen, K.; Shaw, D. E. Atomic-level description of ubiquitin folding. Proc. Natl. Acad. Sci. U.S.A. 2013, 110, 5915-5920.

(11) Kaszuba, K.; Postila, P. A.; Cramariuc, O.; Sarewicz, M.; Osyczka, A.; Vattulainen, I.; Rog, T. Parameterization of the prosthetic redox centers of the bacterial cytochrome bc(1) complex for atomistic molecular dynamics simulations. Theo. Chem. Acc. 2013, 132.

(12) Torshin, I. Y.; Weber, I. T.; Harrison, R. W. Geometric criteria of hydrogen bonds in proteins and identification of 'bifurcated' hydrogen bonds. Protein Eng. 2002, 15, 359-363. 